\title{
Noise Reduction Based on an Fe-Rh Interlayer in Exchange-Coupled Heat-Assisted Recording Media
}

\author{
Christoph Vogler* \\ Faculty of Physics, University of Vienna, Boltzmanngasse 5, 1090 Vienna, Austria \\ Claas Abert, Florian Bruckner, and Dieter Suess \\ Christian Doppler Laboratory for Advanced Magnetic Sensing and Materials, Faculty of Physics, \\ University of Vienna, Boltzmanngasse 5, 1090 Vienna, Austria
}

(Received 9 June 2017; published 10 November 2017)

\begin{abstract}
High storage density and high data rate are two of the most desired properties of modern hard disk drives. Heat-assisted magnetic recording (HAMR) is believed to achieve both. Recording media, consisting of exchange-coupled grains with a high and a low $T_{C}$ part, were shown to have low dc noise- but increased ac noise - compared to hard magnetic single-phase grains like FePt. We extensively investigate the influence of an Fe-Rh interlayer on the magnetic noise in exchange-coupled grains. We find an optimal grain design that reduces the jitter in the down-track direction by up to $30 \%$ and in the off-track direction by up to $50 \%$, depending on the head velocity, compared to the same structures without FeRh. Furthermore, the mechanisms causing this jitter reduction are demonstrated. Additionally, we show that, for short heat pulses and low write temperatures, the switching-time distribution of the analyzed grain structure is reduced by a factor of 4 compared to the same structure without an Fe-Rh layer. This feature could be interesting for HAMR use with a pulsed laser spot and could encourage discussion of this HAMR technique.
\end{abstract}

DOI: 10.1103/PhysRevApplied.8.054021

\section{INTRODUCTION}

The areal storage density (AD) of conventional perpendicular magnetic recording (PMR) has shown a tremendous increase over the decades. Almost $20 \mathrm{yr}$ ago, when the annual $\mathrm{AD}$ growth was about $100 \%$, a limit of $1 \mathrm{Tbit} / \mathrm{in}^{2}$ was predicted for PMR [1]. Indeed, in recent years, the growth rate has significantly slowed down and seems to reach saturation. Current ADs of PMR media are already in the vicinity of $1 \mathrm{Tbit} / \mathrm{in}^{2}$. Alternative approaches like twodimensional magnetic recording and shingled recording were proposed to extend this limit to about $1.5 \mathrm{Tbit} / \mathrm{in}^{2}[2,3]$.

Nevertheless, to reach even higher ADs, a new recording concept is needed. Heat-assisted magnetic recording (HAMR) is expected to be this key technology. Although the basic principle of HAMR was proposed nearly $60 \mathrm{yr}$ ago [4], only recent advances in the development of near-field transducers have allowed fabrication of HAMR heads for high-density recording. HAMR uses the decrease of the coercivity of a ferromagnetic material with temperature. Hence, magnetic grains with a high magnetic anisotropy can be switched, which would not be possible at room temperature. As a

\footnotetext{
*christoph.vogler@univie.ac.at
}

Published by the American Physical Society under the terms of the Creative Commons Attribution 4.0 International license. Further distribution of this work must maintain attribution to the author(s) and the published article's title, journal citation, and DOI. consequence, recording grains can be further scaled down while maintaining their long-term stability. The loss of longterm stability for small structures, known as the superparamagnetic limit, is the bottleneck of PMR. Even more importantly, because of the heat assist, the higher effective field gradient of a HAMR head,

$$
\frac{d H_{\mathrm{eff}}}{d x}=\frac{d\left|H_{\mathrm{ext}}\right|}{d x}+\frac{d H_{c}}{d T} \frac{d T}{d x},
$$

allows us to narrow the bit transitions, ultimately yielding smaller bits.

First working devices with an AD of $1 \mathrm{Tbit} / \mathrm{in}^{2}$ [5] in 2013 and $1.4 \mathrm{Tbit} / \mathrm{in}^{2}$ [6] in 2015 proved the potential of HAMR. Future hard disk drives based on granular media are hoped to reach ADs of up to $4 \mathrm{Tbit} / \mathrm{in}^{2}$ [7-9]. In combination with bit-patterned media, theoretical investigations predict ADs even beyond $10 \mathrm{Tbit} / \mathrm{in}^{2}$ [10].

The downside of the latter study is the low head velocity of $7.5 \mathrm{~m} / \mathrm{s}$ for which the high AD is obtained. For higher data rates, dc noise arises at high temperatures. The reason is the combination of a high thermal gradient of the heat spot and a high $d H_{c} / d T$ gradient near the Curie temperature of the high anisotropy islands used (such as FePt). Both result in a short recording time window $[11,12]$ in which the external field can efficiently reverse the magnetization of the islands [13]. Hence, the switching is not reliable at high data rates. One possible solution for increasing the head velocity without creating problems 


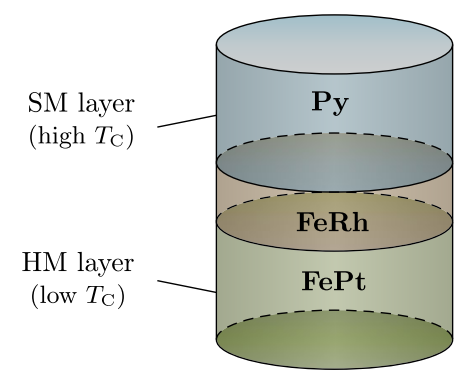

FIG. 1. Schematic illustration of the proposed grain structure.

with dc noise is the use of exchange-coupled grains with a high and a low $T_{C}$ part, thereby lowering the $d H_{c} / d T$ gradient. However, with such grains, ac noise increases, which significantly decreases the maximum AD [14].

In this work, we show how an Fe-Rh layer in the middle of a grain with a high- and a low- $T_{C}$ part $(\mathrm{Py} / \mathrm{FePt}$, for example) can reduce ac noise without leading to dc noise, even for high data rates. At room temperature, FeRh is antiferromagnetic. Above a critical temperature, $T_{\text {jump }}$, it becomes ferromagnetic [15]. The key idea of the proposed structure, as illustrated in Fig. 1, is to use a thin Fe-Rh layer between the soft magnetic (SM) high- $T_{C}$ layer and the hard magnetic (HM) low- $T_{C}$ layer, which controls the exchangecoupling strength between them. In the ferromagnetic phase, well above $T_{\text {jump }}$, we assume full coupling, and towards room temperature the coupling drastically decreases. We show that the proposed grain combines the best of pure HM grains with low ac noise and exchange-coupled grains with low dc noise. Furthermore, we investigate which requirements the Fe-Rh interlayer must fulfill to gain the most from the proposed structure compared to ordinary grains with a high- and a low- $T_{C}$ part.

In contrast to $\mathrm{Fe}-\mathrm{Rh} / \mathrm{HM}$ grains without a soft top layer, in which FeRh is used to produce an exchange-spring effect above $T_{\text {jump }}$ [16], FeRh merely tunes the exchange coupling in the proposed structure. FeRh itself is not required to lower the coercivity of the composite grain. For the read-back process, the additional magnetic moment of the SM layer is a large advantage towards achieving high signal-to-noise ratios even for small grain dimensions. This is also in contrast to the $\mathrm{Fe}-\mathrm{Pt} / \mathrm{Fe}-\mathrm{Rh} / \mathrm{Fe}-\mathrm{Co}$ trilayer structure of Refs. [17,18], which has an in-plane Fe-Co layer whose magnetic moment cannot be used during read back.

\section{METHODS}

To correctly model the magnetization dynamics during the HAMR process, we use a coarse-grained model based on the Landau-Lifshitz-Bloch (LLB) equation [19-26]. Specifically, the stochastic formulation proposed by Evans et al. [27] per

$$
\begin{aligned}
\frac{d \boldsymbol{m}}{d t}= & -\mu_{0} \gamma^{\prime}\left(\boldsymbol{m} \times \boldsymbol{H}_{\mathrm{eff}}\right) \\
& -\frac{\alpha_{\perp} \mu_{0} \gamma^{\prime}}{m^{2}}\left\{\boldsymbol{m} \times\left[\boldsymbol{m} \times\left(\boldsymbol{H}_{\mathrm{eff}}+\boldsymbol{\xi}_{\perp}\right)\right]\right\} \\
& +\frac{\alpha_{\|} \mu_{0} \gamma^{\prime}}{m^{2}} \boldsymbol{m}\left(\boldsymbol{m} \cdot \boldsymbol{H}_{\mathrm{eff}}\right)+\boldsymbol{\xi}_{\|} .
\end{aligned}
$$

is solved. In this equation, $\boldsymbol{m}$ is the reduced magnetization $\boldsymbol{M} / M_{0}$, with the saturation magnetization at zero temperature $M_{0}, \gamma^{\prime}$ is the reduced electron gyromagnetic ratio $\left[\gamma^{\prime}=\left|\gamma_{e}\right| /\left(1+\lambda^{2}\right)\right.$, with $\left.\left|\gamma_{e}\right|=1.76086 \times 10^{11}(\mathrm{~T} \mathrm{~s})^{-1}\right], \mu_{0}$ is the vacuum permeability, and $\alpha_{\|}$and $\alpha_{\perp}$ are dimensionless temperature-dependent longitudinal and transverse damping parameters defined as

$$
\alpha_{\perp}=\left\{\begin{array}{ll}
\lambda\left(1-\frac{T}{T_{C}}\right) & T<T_{C} \\
\alpha_{\|} & T \geq T_{C}
\end{array}, \quad \alpha_{\|}=\lambda \frac{2 T}{3 T_{C}} .\right.
$$

$\lambda$ denotes the coupling of the spin to the heat bath on an atomistic level. The effective magnetic field $\boldsymbol{H}_{\text {eff }}$ comprises the external field, the anisotropy field, and exchange fields. Magnetostatic interactions are not directly included. The stochastic influence of temperature is represented by the longitudinal and perpendicular stochastic fields $\boldsymbol{\xi}_{\|}$and $\boldsymbol{\xi}_{\perp}$, respectively. Both are vectors with Gaussian random numbers with zero mean and a variance per

$$
\left\langle\xi_{\eta, i}(t, \boldsymbol{r}) \xi_{\eta, j}\left(t^{\prime}, \boldsymbol{r}^{\prime}\right)\right\rangle=2 D_{\eta} \delta_{i j} \delta\left(\boldsymbol{r}-\boldsymbol{r}^{\prime}\right) \delta\left(t-t^{\prime}\right),
$$

with the diffusion constants $D_{\eta}$ ( $\eta$ is a placeholder for $\|$ and $\perp$ ), which are derived from the fluctuation-dissipation theorem per

$$
\begin{aligned}
D_{\perp} & =\frac{\left(\alpha_{\perp}-\alpha_{\|}\right) k_{B} T}{\gamma^{\prime} \mu_{0}^{2} M_{0} V \alpha_{\perp}^{2}}, \\
D_{\|} & =\frac{\alpha_{\|} \gamma^{\prime} k_{B} T}{M_{0} V} .
\end{aligned}
$$

Here, $T$ is the temperature, $k_{B}$ is the Boltzmann constant, and $V$ is the discretization volume. The special feature of the coarse-grained LLB model is that each material layer in a magnetic grain is described with a single magnetization vector. Although this coarse-grained model is computationally cheap, it can correctly reproduce the magnetization dynamics of the same system with an atomistic discretization. Please refer to Ref. [28] for more details.

\section{A. Simulation setup of the recording head}

We analyze the switching probabilities of continuous HAMR in the following. This means that a constant laser pulse with a write temperature of $T_{\text {write }}=715 \mathrm{~K}$ in the spot center and a full width at half maximum (FWHM) of $20 \mathrm{~nm}$ is assumed to move over a recording medium. The heat 
pulse has a Gaussian shape in space-and thus also in time. Thermal spot sizes of recent HAMR prototypes are about $50 \mathrm{~nm}[5,6]$. Computer simulations of a lollipop near field transducer [29] show, depending on the medium, that a FWHM $<40 \mathrm{~nm}$ is feasible $[7,30]$. Nevertheless, further research will be required to reduce the FWHM down to about $20 \mathrm{~nm}$, in order to achieve an AD beyond 5 Tbit/in ${ }^{2}$.

Furthermore, we assume the inductive write head will produce a spatially uniform field with a strength of $\mu_{0} H_{\text {ext }}=0.8 \mathrm{~T}$. We choose a typical write frequency of $1 \mathrm{GHz}$. The easy axis of the grains and the external field enclose an angle of $6^{\circ}$. With this setup, we investigate the switching behavior of isolated grains with an initial magnetization state that points in the negative- $z$ direction, hereafter referred to as magnetization down. Depending on the off-track position of a grain in the medium, the maximum temperature of the arriving heat pulse differs per

$$
T_{\max }(y)=\left(T_{\text {write }}-T_{\min }\right) e^{-\left(y^{2} / 2 \sigma^{2}\right)}+T_{\text {min }},
$$

with $y$ representing the off-track position, $T_{\min }=270 \mathrm{~K}$, and

$$
\sigma=\frac{\mathrm{FWHM}}{\sqrt{8 \ln (2)}}
$$

The maximum temperature of the heat pulse is not dependent on the down-track direction, but the temporal shift between the maximum of the heat pulse and the center of the write pulse varies. To illustrate the situation for different bit positions, Fig. 2 displays the temporal evolution of the heat pulse and the external field for a write pattern of 0001000 . (a)

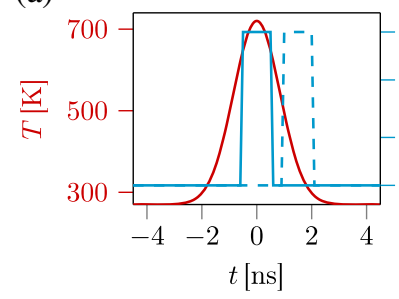

(b)

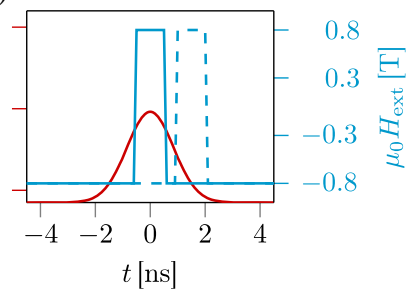

FIG. 2. Illustration of the temporal evolution of the applied heat pulse and the external magnetic field for a write pattern of 0001000 for different grain positions. A head velocity of $10 \mathrm{~m} / \mathrm{s}$ is assumed. (a) Grain at the center of the track (off-track position of $y=0 \mathrm{~nm}$ ) and a down-track position of $x=0 \mathrm{~nm}$ (the solid blue line) and $x=15 \mathrm{~nm}$ (the dashed blue line), respectively. (b) Grain at the same down-track positions as in (a) but with an off-track position of $y=10 \mathrm{~nm}$, yielding a lower maximum temperature of the heat pulse.

\section{B. Composition of the recording grains}

The grains in the investigated media have a diameter of $5 \mathrm{~nm}$ and a thickness of $10 \mathrm{~nm}$. We compare three grain types:

(a) single-phase grains with the material property $\mathrm{HM}$ from Table I,

(b) exchange-coupled $\mathrm{SM} / \mathrm{HM}$ grains consisting of $50 \%$ SM (SM1 or SM2) and 50\% HM material, and

(c) $\mathrm{SM} / \mathrm{Fe}-\mathrm{Rh} / \mathrm{HM}$ grains with the same composition as (b) but with an Fe-Rh interlayer between the SM and HM layers.

The HM material referred to in Table I is very similar to pure FePt, except for the reduced Curie temperature. A decrease of $T_{C}$, while maintaining high anisotropy, can be obtained by adding a few-percent concentration of $\mathrm{Cu}$ or $\mathrm{Ni}$, as demonstrated in Refs. [31-33]. The SM layers have properties similar to those of permalloy (Py) with an enhanced damping constant. Although this assumption seems risky, Bailey et al. [34] showed how to significantly increase the damping constant in Py while maintaining its soft magnetic character by doping with low concentrations of $\mathrm{Tb}$.

As mentioned in Sec. I, FeRh is antiferromagnetic at room temperature and becomes ferromagnetic above a critical temperature $T_{\text {jump. }}$. In the proposed structure, a thin Fe-Rh layer between the SM and the HM layer is used to control the exchange-coupling strength between them. In our model, we do not compute the detailed magnetization dynamics of the Fe-Rh interlayer: we consider only the effected modulation of the exchange coupling, as demonstrated in Fig. 4(b). At high temperatures well above $T_{\text {jump}}$, the SM and HM layers are fully coupled and, at room temperature, the exchange coupling reaches its minimum value.

\section{Stabilization of the soft layer}

In the proposed $\mathrm{SM} / \mathrm{Fe}-\mathrm{Rh} / \mathrm{HM}$ structure, we must ensure that the magnetization of the SM layer is oriented in the correct direction (parallel to that of the $\mathrm{HM}$ layer) during read back. Before discussing the recording performance of the introduced grains, we want to analyze the requirements under which the proposed

TABLE I. Zero-temperature magnetic properties of the soft magnetic (SM) and hard magnetic (HM) materials used. $K_{1}$ is the anisotropy constant, $M_{S}$ the saturation magnetization, $\lambda$ the spinbath coupling strength, and $T_{C}$ the Curie temperature.

\begin{tabular}{llll}
\hline \hline & HM & SM1 & SM2 \\
\hline$K_{1}\left(\mathrm{MJ} / \mathrm{m}^{3}\right)$ & 6.6 & 0.0 & 0.0 \\
$\mu_{0} M_{S}(\mathrm{~T})$ & 1.43 & 1.43 & 1.00 \\
$\lambda$ & 0.1 & 1.0 & 1.0 \\
$T_{C}(\mathrm{~K})$ & 537 & 822 & 849 \\
\hline \hline
\end{tabular}


$\mathrm{SM} / \mathrm{Fe}-\mathrm{Rh} / \mathrm{HM}$ structures show this parallel alignment, and thus a high read-back signal, due to the high moment of the SM part, is ensured.

In equilibrium, the exchange field of the HM layer, acting on the SM layer, must be larger than the sum of the stray fields of all neighboring grains and the stray field of the bottom HM layer. This means that the exchange coupling between the SM layer and the HM layer must not completely vanish at room temperature. As a consequence, it is a requirement that the $\mathrm{Fe}-\mathrm{Rh}$ interlayer must not become a perfectly compensated antiferromagnet. To estimate the required room-temperature coupling strength, we calculate the stray field of all neighboring grains and the bottom HM layer for a worstcase scenario. In this scenario, all grains in an area of $50 \times 50 \mathrm{~nm}^{2}$ of a granular medium are assumed to be magnetized in the up direction, in an attempt to reverse the SM layer. The surface of the medium consists of $83 \%$ grains and $17 \%$ grain boundary, which corresponds to an average boundary thickness of $0.5 \mathrm{~nm}$ for grains with an average diameter of $5 \mathrm{~nm}$. We generate 1000 different realizations of the described sector via Voronoi tessellation and compute the average stray field in the top part of the center grain with MAGNUM.FE [35]. Specifically, a hybrid finite element-boundary element method based on the Fredkin-Koehler approach [36] is used for the computation of the stray field. Figure 3(a) demonstrates a slice of one realization of the medium. The colors are used to distinguish between the HM and SM parts of the grains. A histogram of the stray field in the top (SM1) part of the center grain, which is highlighted in Fig. 3(a), is illustrated in Fig. 3(b). The resulting maximum and average demagnetizing fields of both $\mathrm{HM} / \mathrm{SM}$ structures are given in Table II. According to Ref. [28], the intergrain exchange field of the HM part acting on the SM part can be computed per (a)

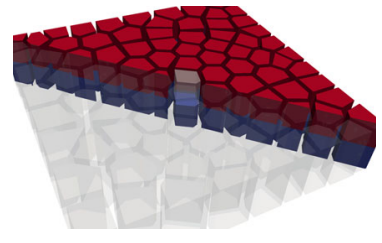

(b)

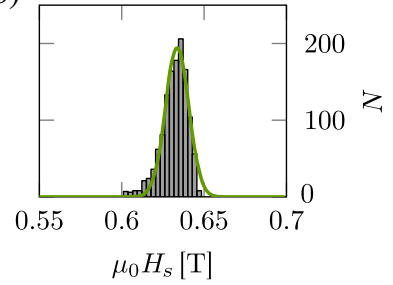

FIG. 3. (a) Slice through a medium with an area of $50 \times 50 \mathrm{~nm}^{2}$, consisting of SM/HM grains with an average diameter of $5 \mathrm{~nm}$ and a thickness of $10 \mathrm{~nm}$. Colors are used to distinguish between the HM and SM parts of the grains. The stray field in the upper, highlighted SM part of the center grain is evaluated. (b) Stray field distribution in the upper, highlighted SM1 part of the center grain, produced by all neighboring grains and the lower HM part of the center grain. The distribution is computed from 1000 different media realizations.
TABLE II. Average and maximum stray field in the soft magnetic part of the center grain, as illustrated in Fig. 3. $A_{\text {iex }}(0)$ denotes the full exchange coupling between the soft and hard magnetic layers at zero temperature.

\begin{tabular}{lccc}
\hline \hline Grain & $A_{\text {iex }}(0)$ & $\mu_{0} H_{s, \text { av }}$ & $\mu_{0} H_{s, \text { max }}$ \\
\hline SM1/HM & $25.75 \mathrm{pJ} / \mathrm{m}$ & $634 \mathrm{mT}$ & $648 \mathrm{mT}$ \\
SM2/HM & $25.75 \mathrm{pJ} / \mathrm{m}$ & $604 \mathrm{mT}$ & $614 \mathrm{mT}$ \\
\hline \hline & & \\
& $H_{\text {iex,SM }}(T)=\frac{2 A_{\text {iex }}(T)}{a t \mu_{0} M_{S, S M}(T)}$, &
\end{tabular}

where $a$ is the atomistic lattice constant, $t$ the layer thickness, and $M_{S, S M}(T)$ the saturation magnetization of the SM layer at the temperature $T$. The obtained reduction of the intergrain exchange field acting on the SM1 layer with decreasing coupling strength is illustrated in Fig. 4(a). To ensure that the magnetization of the SM layer points in the same direction as the HM layer magnetization, we must claim that $H_{\mathrm{iex}, \mathrm{SM}} \geq H_{s, \max }$ is valid at room temperature. This requirement holds if the remaining exchange coupling in the $\mathrm{SM} / \mathrm{Fe}-\mathrm{Rh} / \mathrm{HM}$ structure at room temperature is larger than $r_{\mathrm{ex}} A_{\text {iex,full }}(0)$. Here, $r_{\mathrm{ex}}$ is the remaining fraction of exchange coupling, with regard to full coupling at zero temperature, which is required to stabilize the corresponding SM layer at room temperature [see Fig. 4(b)]. Hence, $r_{\mathrm{ex}}$ is a measure for the required remaining ferromagnetic moment of the $\mathrm{Fe}-\mathrm{Rh}$ layer at room temperature, which depends on the composition of the whole grain.

The above considerations suggest that the Fe-Rh interlayer used must meet some requirements, like a remaining ferromagnetic moment at room temperature. It has been demonstrated that the remaining room-temperature ferromagnetic moment of $\mathrm{FeRh}$ depends on the annealing temperature [37,38], on the composition [39], on the film thickness, and on the extent of atomic ordering [40]. Hence, (a)

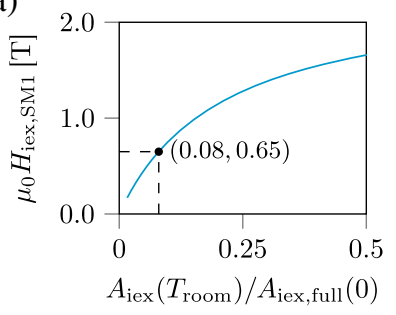

(b)

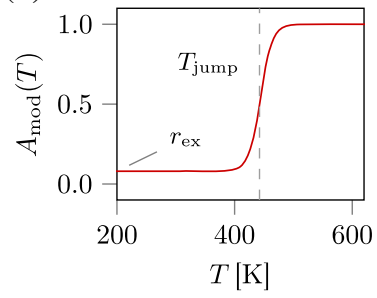

FIG. 4. (a) Intergrain exchange field of a HM layer acting on a SM1 layer as function of the reduction of the intergrain exchange constant. The exchange constant is normalized to the full exchange at zero temperature. (b) Modulation function of the intergrain exchange of an $\mathrm{Fe}-\mathrm{Rh}$ interlayer in a $\mathrm{SM} 1 / \mathrm{Fe}-\mathrm{Rh} 2 / \mathrm{HM}$ structure (see Table III). $r_{\mathrm{ex}}$ denotes the remaining fraction of exchange at low temperatures due to a remaining net magnetic moment of FeRh and $T_{\text {jump }}$ denotes the critical jump temperature of the first-order phase transition in FeRh. 
TABLE III. Parameters, taken from Eqs. (9) and (10), defining the intergrain exchange-modulation functions of $\mathrm{FeRh}$ in three different grain structures.

\begin{tabular}{lccccc}
\hline \hline Grain & $T_{\text {jump }}(\mathrm{K})$ & $\Delta T(\mathrm{~K})$ & $r_{\text {ex }}(\%)$ & $\alpha$ & $\beta$ \\
\hline SM1/Fe-Rh1/HM & 442 & 20 & 0.0 & 0.50 & 0.50 \\
SM1/Fe-Rh2/HM & 444 & 20 & 8.0 & 0.46 & 0.54 \\
SM2/Fe-Rh3/HM & 466 & 20 & 6.7 & 0.47 & 0.53 \\
\hline \hline
\end{tabular}

for small films, it seems to be adjustable. As shown in the following text, the critical temperature of the first-order phase transition $T_{\text {jump }}$ is also an important quantity for the proposed structure. $T_{\text {jump }}$ is known to be tunable in a wide temperature range $[16,41,42]$. To quantify the exchangemodulation function of the Fe-Rh layer, we use [43]

$$
\frac{A_{\text {iex }}(T)}{A_{\text {iex }}(0)}=\alpha \tanh \left(\frac{T-T_{\text {jump }}}{\Delta T}\right)+\beta .
$$

Here, $\Delta T$ denotes the width of the transition, $\alpha$ is a scaling parameter, and $\beta$ is an offset parameter. According to measurements of Fe-Rh thin films in Refs. [40,43], we use a typical value of $\Delta T=20 \mathrm{~K}$. The scaling and offset parameters are computed from the requirement of the remaining exchange-coupling fraction $r_{\mathrm{ex}}$ well below $T_{\text {jump }}$ per

$$
\begin{aligned}
& \alpha=\frac{1-r_{\mathrm{ex}}}{1-\tanh \left(-\frac{T_{\mathrm{jump}}}{\Delta T}\right)} \\
& \beta=r_{\mathrm{ex}}-\alpha \tanh \left(-\frac{T_{\mathrm{jump}}}{\Delta T}\right) .
\end{aligned}
$$

The parameters for all of the examined SM/Fe-Rh/HM structures are given in Table III.

Note that there are many requirements for the Fe-Rh layer in the proposed structure. It is not clear if all of them can be fulfilled. However, in this work, we aim to investigate the potential benefits of $\mathrm{SM} / \mathrm{Fe}-\mathrm{Rh} / \mathrm{HM}$ grains in HAMR. Hence, we assume the desired properties. Furthermore, the presented structures can serve as design guidelines for experimental research on FeRh.

\section{RESULTS}

\section{A. Recording performance}

By means of the coarse-grained LLB model introduced in Sec. II and the HAMR setup of Sec. II A, we simulate footprints of the HAMR head on recording media consisting of the grain types described in Sec. II B. Three different head velocities are examined as displayed in Fig. 5. It is assumed that a grain is located at each phase point of Fig. 5, and 128 write trials are computed for each position. The switching probability $P$ is finally obtained from the fraction of successfully reversed grains. In the case of bit-patterned media, the presented phase diagrams directly determine the switching probability of bits and, in the case of granular media, they can be interpreted as average footprints of the head. Hence, for both kind of media, conclusions can be drawn.

Figure 5(a) shows narrow transitions for a head velocity of $7.5 \mathrm{~m} / \mathrm{s}$ and pure HM grains. For bit-patterned media consisting of the same HM grains Ref. [10] demonstrated a maximum areal storage density of about $13 \mathrm{Tbit} / \mathrm{in}^{2}$ if a shingled write schema is used. Although the transitions remain narrow at higher head velocities, dc noise becomes a serious problem. The reason is the combination of a high thermal gradient and a high head velocity, both of which reduce the recording time window of the write process. According to Ref. [13], the effective recording time window (ERTW) is defined per

$$
\mathrm{ERTW}_{\uparrow}=\left[t\left(T_{C}\right), t\left(T_{f}\right)\right] \cap\left[t_{\uparrow, \text { start }}, t_{\uparrow, \text { final }}\right],
$$

where $T_{C}$ is the Curie temperature and $T_{f}$ is the freezing temperature. At $T_{f}$, the coercive field of a grain becomes lower than the available write field. Hence, Eq. (11) depicts the intersection of the time interval during which the external field points in the write direction $\left[t_{\uparrow, \text { start }}, t_{\uparrow, \text { final }}\right]$ and the time interval $\left[t\left(T_{C}\right), t\left(T_{f}\right)\right]$, during which the coercive field of the grains is lower than the write field. Only during the ERTW can the external field efficiently act on the magnetization of the particles. If the recording time window is smaller than a threshold value, the switching process is not reliable, yielding dc noise (see Ref. [13]), in the case of the pure HM grain and $v=20 \mathrm{~m} / \mathrm{s}$.

The exchange spring SM/HM structure significantly reduces dc noise even for higher head velocities, at the expense of a much broader transition area due to the increased ac noise, as Fig. 5(b) indicates. This is the case because of the lower $d H_{c} / d T$ gradient near the Curie temperature, which enlarges the recording time window and thus reduces dc noise (but increases ac noise). This means, in principle, that higher data rates are possible but the distance between neighboring bits in bit-patterned media or the transition jitter in granular media increases, resulting in lower maximum areal densities (see Ref. [14]).

To quantify the transition width in the down-track direction the switching probabilities along the center track $(y=0$ in Fig. 5) are fitted with the cumulative distribution function of the normal distribution, as demonstrated in Fig. 6. The obtained standard deviation $\sigma$ is a measure for the transition jitter. The same fitting procedure along the marked dashed lines in Fig. 5 is performed to extract the transition width in the off-track direction. Table IV summarizes the jitter values for all investigated grains and for head velocities of 7.5 and $20 \mathrm{~m} / \mathrm{s}$, respectively.

As expected, the transition jitter of the SM/HM structures is significantly higher than in the pure HM grains in both directions. The proposed SM1/Fe-Rh2/HM structure 
(a)

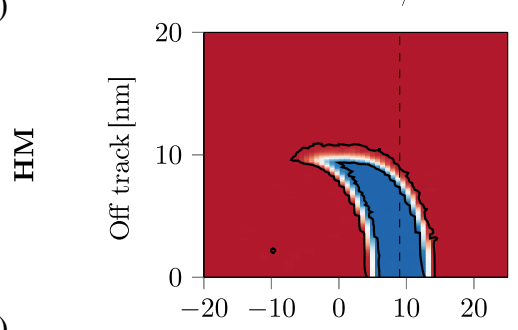

(b)

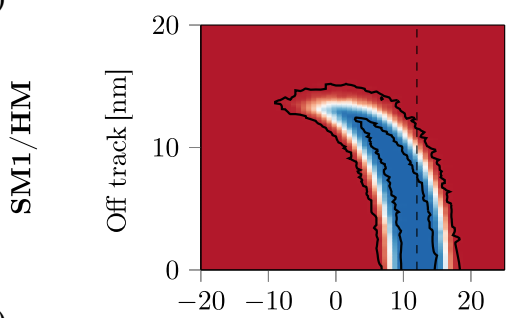

(c)

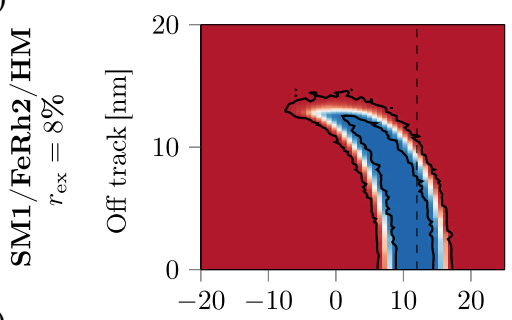

(d)

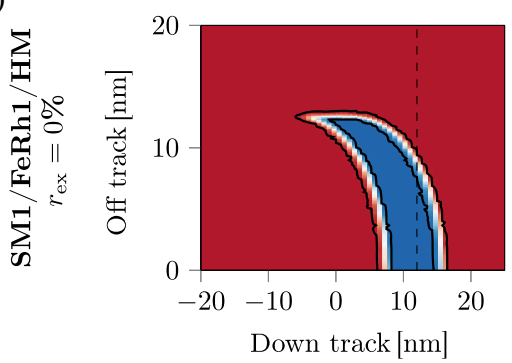

$v=10 \mathrm{~m} / \mathrm{s}$
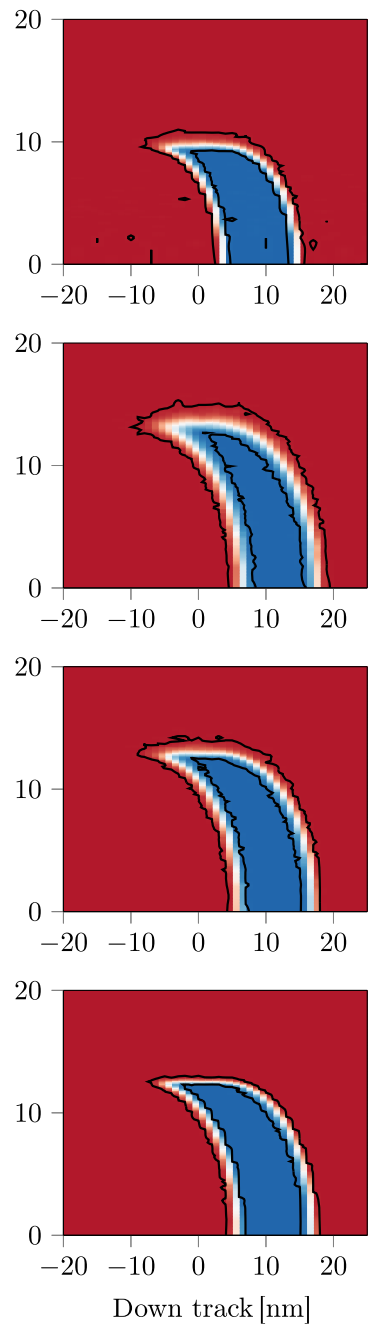
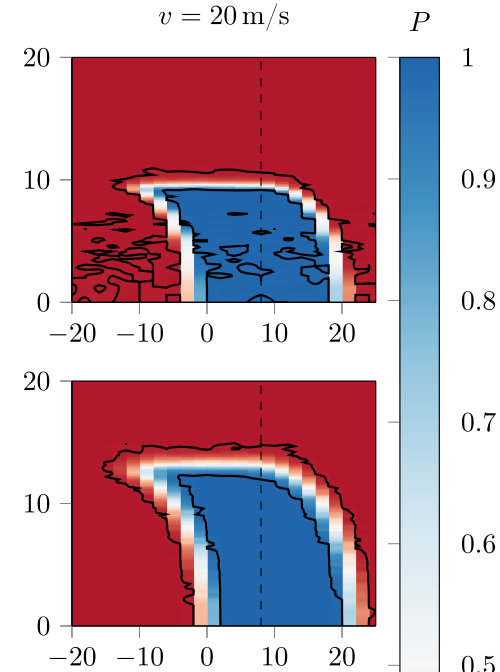

0.7

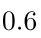

0.5
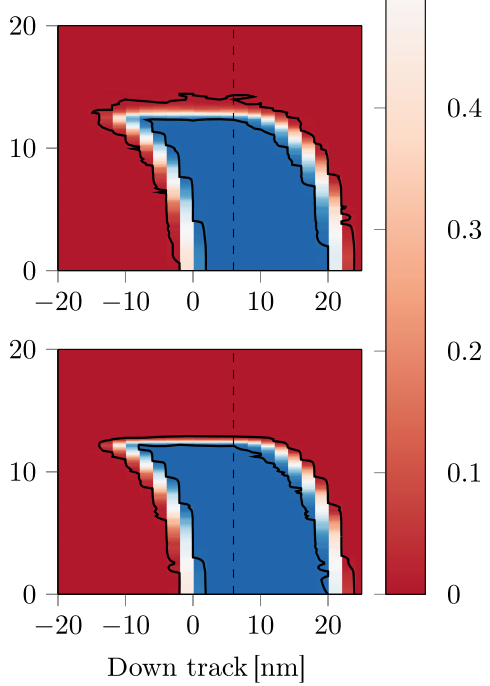

FIG. 5. Footprint of a HAMR head for three different velocities, written for (a) pure HM grains, (b) exchange-coupled SM1/HM grains, (c) SM1/Fe-Rh2/HM grains, and (d) SM1/Fe-Rh1/HM grains. The color code illustrates the switching probability $P$ of a grain located at the corresponding position. $P$ is computed from 128 switching trajectories, which try to reverse the magnetization from an initial down to an up direction. A FWHM of $20 \mathrm{~nm}$ and an external field strength of $0.8 \mathrm{~T}$ is assumed for all phase points and grain types. The contour lines separate the areas with $0 \%$ and $100 \%$ switching probability. Along the vertical dashed lines, the off-track jitter displayed in Table IV is computed.

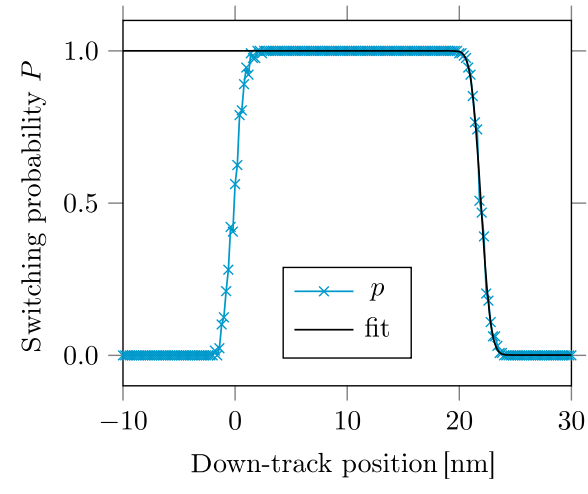

FIG. 6. Switching probability of a SM1/HM grain for a head velocity of $20 \mathrm{~m} / \mathrm{s}$ along the center track taken from Fig. 5(b). The transition is fitted with the cumulative distribution function of the normal distribution to extract a measure for the transition width. with a remaining ferromagnetic moment in the $\mathrm{Fe}-\mathrm{Rh}$ interlayer with $r_{\mathrm{ex}}=8 \%$ [for a definition of $r_{\mathrm{ex}}$, see Eqs. (9) and (10), as well as Fig. 4(b)] shows a reduction of down-track jitter of about $20 \%-25 \%$ for both examined head velocities (see Table IV) compared to ordinary SM1/HM grains. As Fig. 5(c) indicates, no dc noise appears independent of the head velocity. The off-track jitter gain is even higher, with about $30 \%$ in the case of $v=7.5 \mathrm{~m} / \mathrm{s}$ and $50 \%$ for $v=20 \mathrm{~m} / \mathrm{s}$. Very similar values are obtained for $\mathrm{SM} 2 / \mathrm{Fe}-\mathrm{Rh} 3 / \mathrm{HM}$ grains with $r_{\text {ex }}=6.7 \%$. SM2/HM grains show slightly higher jitter values compared to SM1/HM structures with a higher saturation magnetization in the soft magnetic part, but the jitter reduction is almost the same for both analyzed grain types, if a suitable Fe-Rh interlayer is used. Table IV demonstrates that the jitter reduction can be notably 
TABLE IV. Transition jitter in the down-track and off-track directions for various grain types and head velocities. The jitter reductions of the $\mathrm{SM} / \mathrm{Fe}-\mathrm{Rh} / \mathrm{HM}$ structures refer to the corresponding ordinary SM/HM grains.

\begin{tabular}{|c|c|c|c|c|c|c|c|c|c|}
\hline \multirow[b]{2}{*}{ Grain } & \multirow[b]{2}{*}{$r_{\mathrm{ex}}(\%)$} & \multicolumn{4}{|c|}{$v=7.5 \mathrm{~m} / \mathrm{s}$} & \multicolumn{4}{|c|}{$v=20 \mathrm{~m} / \mathrm{s}$} \\
\hline & & $\sigma_{\text {down }}(\mathrm{nm})$ & $\sigma_{\mathrm{off}}(\mathrm{nm})$ & $\sigma_{\text {down,red }}(\%)$ & $\sigma_{\text {off,red }}(\%)$ & $\sigma_{\text {down }}(\mathrm{nm})$ & $\sigma_{\text {off }}(\mathrm{nm})$ & $\sigma_{\text {down,red }}(\%)$ & $\sigma_{\text {off,red }}(\%)$ \\
\hline $\mathrm{HM}$ & $\ldots$ & 0.20 & 0.27 & $\ldots$ & $\ldots$ & 0.39 & 0.23 & $\ldots$ & $\ldots$ \\
\hline SM1/HM & $\cdots$ & 0.55 & 0.74 & $\ldots$ & $\cdots$ & 0.70 & 0.49 & $\ldots$ & $\cdots$ \\
\hline $\mathrm{SM} 2 / \mathrm{HM}$ & $\cdots$ & 0.62 & 0.83 & $\ldots$ & $\ldots$ & 0.70 & 0.51 & $\ldots$ & $\ldots$ \\
\hline $\mathrm{SM} 1 / \mathrm{Fe}-\mathrm{Rh} 1 / \mathrm{HM}$ & 0.0 & 0.33 & 0.33 & -39.19 & -55.07 & 0.52 & 0.09 & -25.86 & -81.68 \\
\hline $\mathrm{SM} 1 / \mathrm{Fe}-\mathrm{Rh} 2 / \mathrm{HM}$ & 8.0 & 0.41 & 0.51 & -24.23 & -30.83 & 0.55 & 0.25 & -20.75 & -49.26 \\
\hline SM2/Fe-Rh3/HM & 6.7 & 0.45 & 0.54 & -27.13 & -35.01 & 0.50 & 0.27 & -27.88 & -47.83 \\
\hline
\end{tabular}

increased if the Fe-Rh interlayer is a perfect antiferromagnet at room temperature $\left(r_{\mathrm{ex}}=0 \%\right)$. The reason will become clear in Sec. III B. This structure is investigated to show the maximum possible gain of the interlayer. It cannot be used in a real device due to the decoupled soft magnetic layer at room temperature, which would deteriorate the read-back signal.

In a nutshell, the presented footprints of a HAMR head and the corresponding jitter calculations show that a medium consisting of well-designed $\mathrm{SM} / \mathrm{Fe}-\mathrm{Rh} / \mathrm{HM}$ grains can significantly reduce the transition jitter in both the down-track and the off-track direction while maintaining a low dc error. At high temperatures, the structure behaves like an ordinary SM/HM grain because, well above $T_{\text {jump }}$, the exchange coupling between SM and HM layer has full strength. Hence, dc noise does not arise even at high head velocities. Although the transitions in pure HM grains are narrower, $\mathrm{SM} / \mathrm{Fe}-\mathrm{Rh} / \mathrm{HM}$ grains could be a good compromise for intermediate to high $\mathrm{AD}$, if the requirements for the Fe-Rh interlayer can be met.

\section{B. Origin of jitter reduction}

To understand the reason for the reduction of the transition jitter presented in Sec. III A, the dependence of the freezing temperature $T_{f}$ on the intergrain exchange coupling $A_{\text {iex }}$ between the SM and HM layers must be analyzed. For this purpose, we compute the temperaturedependent coercivity of the SM/HM grains for various values of $A_{\text {iex }}$ by means of Eq. (2). Exemplarily, Fig. 7(a) illustrates the temperature-dependent coercivity of a $\mathrm{SM} 1 / \mathrm{Fe}-\mathrm{Rh} / \mathrm{HM}$ grain with full intergrain exchange coupling $(25.75 \mathrm{pJ} / \mathrm{m})$. To obtain $H_{c}$ at each temperature, repeated easy-axis hysteresis loops are simulated by means of the stochastic LLB equation. Each hysteresis loop is simulated at a constant temperature and a sweep rate of $100 \mathrm{mT} / \mathrm{ns}$. Statistics of 100 loops yield values of the average coercivity and the corresponding standard deviation. The freezing temperature can be evaluated as the temperature at which $H_{c}=H_{\text {ext }}$. Figure 7(b) displays $T_{f}$ for both investigated SM/HM structures and for various intergrain exchange couplings. Both grain types show the same qualitative behavior. $T_{f}$ is almost constant over a wide range of exchange couplings. Only for very small couplings does the freezing temperature increase. The minimum value of $T_{f}$ differs based on the saturation magnetization of the SM part. This dependence of the coercivity on the intergranular exchange is well known in the case of thin exchange-coupled structures [44-46].

The reduction of the off-track jitter in Sec. III A can now be understood by the increase of $T_{f}$ with small exchange couplings. To make this clear, Fig. 8 again shows the footprint of a HMAR head with $v=20 \mathrm{~m} / \mathrm{s}$ written on SM1/HM grains [see also Fig. 5(b)]. In this plot, the offtrack direction is replaced by the maximum temperature of the heat pulse which reaches the grain [see Eq. (6)]. Additionally, the modulation function of the exchange coupling of Fig. 4(b) is displayed as an overlay. The $T_{\text {jump }}$ of the Fe-Rh interlayer is slightly above the minimum freezing temperature for the full coupling $T_{f, \min }$. As a consequence, at the off-track edge of the footprint, the exchange coupling is already small enough that the freezing temperature of the structure increases [see Fig. 7(b)]. Hence, the heat assist is too low to switch the grains and the border of the transition shifts to higher temperatures. At $T_{\text {jump }}, 50 \%$ of the full exchange coupling is available. Based on the findings of Fig. 7(b), the SM1/Fe-Rh/HM structure still has the full exchange-spring effect, and the footprint does

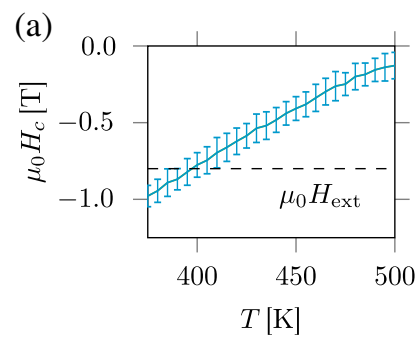

(b)

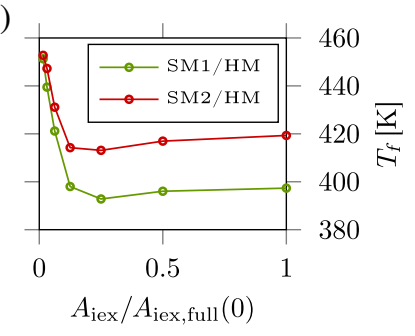

FIG. 7. (a) Coercivity as a function of temperature for a SM1/HM grain. Each data point (value and standard deviation) is obtained from 100 hysteresis loops at a constant temperature and with a sweep rate of $100 \mathrm{mT} / \mathrm{ns}$. (b) The freezing temperature $T_{f}$ versus the exchange coupling between the soft magnetic and the hard magnetic layer for SM1/HM and SM2/HM grains, if an external field of $0.8 \mathrm{~T}$ is applied. The exchange coupling is normalized to the full interaction strength at zero temperature. 


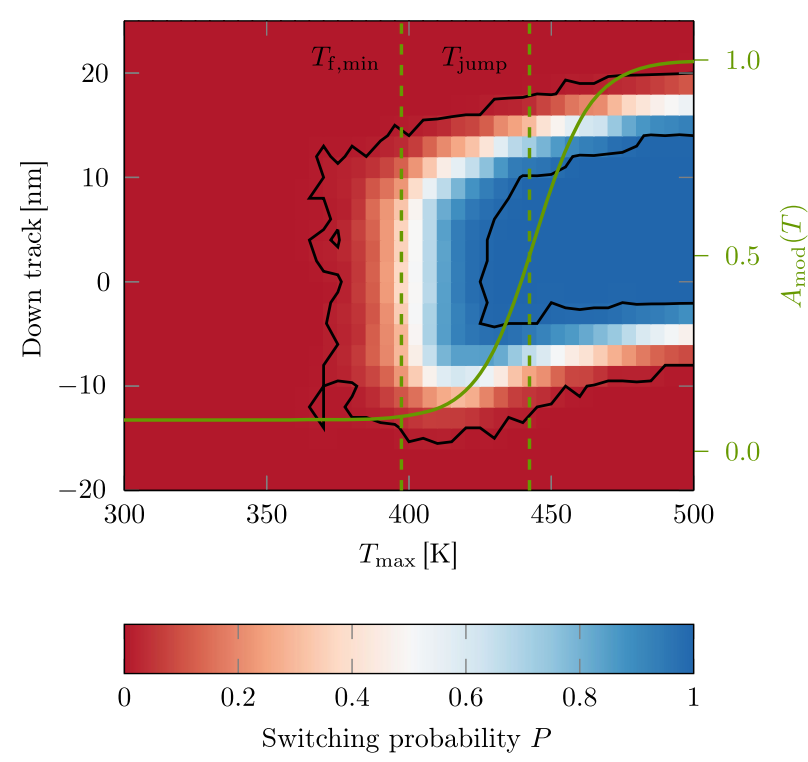

FIG. 8. Footprint of SM1/HM grains and a head velocity of $20 \mathrm{~m} / \mathrm{s}$ taken from Fig. 5(b). The off-track axis is replaced with the maximum temperature of the heat pulse, arising at the corresponding off-track position of the grains. The intergrain exchange modulation function of Fig. 4(b) is plotted as an overlay.

not change compared to the ordinary SM1/HM structure. This means that the width of the transition becomes smaller, which is confirmed by the off-track jitter reduction displayed in Table IV.

The same mechanism of increasing $T_{f}$ at low temperatures or weak exchange coupling is also responsible for the jitter reduction in the down-track direction. During cooling of a grain, the exchange interaction decreases from the full to the minimum coupling, and $T_{f}$ thus increases. Based on Eq. (11), the ERTW becomes smaller as a consequence. A fast-decreasing ERTW directly implies a limitation of the transition area, and thus a jitter reduction. Nevertheless, the down-track jitter will not vanish even if the phase transition of the $\mathrm{Fe}-\mathrm{Rh}$ layer becomes infinitely sharp $[\Delta T \rightarrow 0$ in Eq. (9)]. For $\Delta T=0$, the freezing temperature of a $\mathrm{SM} / \mathrm{Fe}-\mathrm{Rh} / \mathrm{HM}$ structure suddenly increases to that of a pure HM grain at $T_{\text {jump. As a }}$ consequence, the recording time window does not vanishit reduces to that of a pure HM structure. Hence, the down-track jitter reduces, at most, to that of a pure HM grain.

Note that the jitter reduction increase strongly depends on the maximum increase of $T_{f}$ at low temperatures. Hence, it is clear that SM1/Fe-Rh1/HM grains with $r_{\mathrm{ex}}=0 \%$ show a larger jitter reduction. However, $r_{\mathrm{ex}}$ is limited, as discussed in Sec. II C. In these considerations, the stray field of granular media is analyzed. For bitpatterned media with larger spacings between the individual islands, the requirements on $r_{\mathrm{ex}}$ may be relaxed. For $\mathrm{Fe}-\mathrm{Rh} / \mathrm{HM}$ without an additional soft layer [16], the mechanisms should be similar and larger jitter reductions can be expected. Nevertheless, such structures have problems during read back due to the vanishing magnetic moment of $\mathrm{Fe}-\mathrm{Rh}$ at room temperature.

From the above considerations, we see how important the proper design of the $\mathrm{SM} / \mathrm{Fe}-\mathrm{Rh} / \mathrm{HM}$ grains is. For both analyzed structures, a clever choice of $T_{\text {jump }}$ and $r_{\mathrm{ex}}$ yields very similar jitter reductions. On the other hand, there exists some tolerance for the production of the Fe-Rh interlayer if the soft magnetic layer is adapted.

\section{Low soft-layer damping}

Since high damping in the SM layer might require a significant research effort on soft magnetic alloys, we analyze in this section how the demonstrated jitter reduction is influenced if the damping is reduced. For this reason, we compare both the off-track jitter and the down-track jitter of a SM1/HM and a SM1/Fe-Rh2/HM grain with $\lambda=0.1$ in the soft layer. For a head velocity of $v=20 \mathrm{~m} / \mathrm{s}$, Table $\mathrm{V}$ displays the resulting jitter values. The Fe-Rh interlayer still reduces the jitter in both directions, but not as much as in the case of high damping in the SM1 layer. The reason is that the maximal shift of the freezing temperature $\Delta T_{f}$ from full coupling at high temperatures $T_{f, \min }$ to minimal coupling at room temperature $T_{f, \max }\left(r_{\mathrm{ex}}=8 \%\right)$ is reduced by $29 \%$ compared to the case with $\lambda=1$ in the soft layer. Obviously, the exchangespring effect of the $T_{f \text {,min }}$ reduction weakens with lower damping. As a consequence, the narrowing of the transition in the off-track direction, as described in Sec. III B, decreases, resulting in a decreased off-track jitter reduction. The smaller $\Delta T_{f}$ also implies a slower change of $T_{f}$ with temperature. This effect can explain the decreased jitter reduction in the down-track direction because, with a slower varying freezing temperature, the ERTW also decreases slower. Additionally, our simulations show that the switching-time distribution for a SM1/HM grain increases with a lower damping in the soft part. This is a reason for the significantly larger absolute down-track jitter compared to a HM/SM1 grain with $\lambda=1$ and also influences the possible jitter reduction.

In a nutshell, the presented results and considerations show that the basic mechanisms, which are responsible for the jitter reduction due to an Fe-Rh interlayer, do not change for smaller damping in the soft layer. Nevertheless,

TABLE V. Transition jitter in the down-track and off-track directions of a SM1/HM and a SM1/Fe-Rh2/HM grain with $\lambda=0.1$ in the soft layer. The used head velocity is $20 \mathrm{~m} / \mathrm{s}$.

\begin{tabular}{lccc}
\hline \hline Grain & $r_{\text {ex }}(\%)$ & $\sigma_{\text {down }}$ & $\sigma_{\text {off }}$ \\
\hline SM1/HM & $\ldots$ & $1.17 \mathrm{~nm}$ & $0.37 \mathrm{~nm}$ \\
SM1/Fe-Rh2/HM & 8.0 & $1.05 \mathrm{~nm}$ & $0.23 \mathrm{~nm}$ \\
& & $-9.71 \%$ & $-36.82 \%$ \\
\hline \hline
\end{tabular}


the lower jitter reductions and, above all, the large downtrack jitter of SM1/HM and SM1/Fe-Rh2/HM structures with lower damping in the soft layer encourage the effort to produce high-damping materials.

\section{Short heat pulses}

Another interesting aspect of the proposed SM/Fe-Rh/HM structure arises if heat pulses, shorter than the field pulse duration, are used to assist the write process. In such a situation, the external field can be assumed to be constant during the write process. We further assume that $T_{\max }$ is smaller than the Curie temperature of the HM layer. Hence, the magnetization reversal takes place during heating of the grain and not during cooling because the particle never becomes paramagnetic. Under these circumstances, the switching-time distribution of $\mathrm{SM} / \mathrm{Fe}-\mathrm{Rh} / \mathrm{HM}$ grains is significantly shorter than that of the corresponding ordinary $\mathrm{SM} / \mathrm{HM}$ grains, as illustrated in Fig. 9(c). Here, the switching time of 50000 switching trajectories with $T_{\max }=500 \mathrm{~K}$ and a pulse duration of $\sigma_{\text {pulse }}=140 \mathrm{ps}$ are evaluated for three grain types. The last zero crossing of the $z$ component of the magnetization of each layer determines the corresponding switching time. The standard
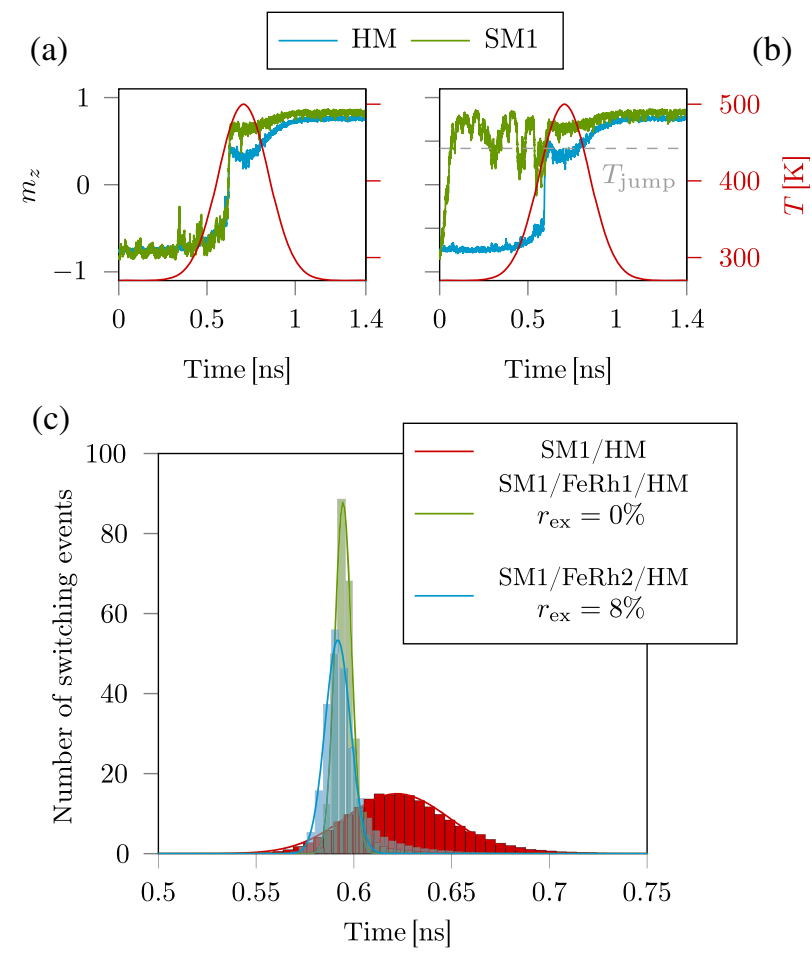

FIG. 9. Switching trajectories of soft and hard magnetic layers in (a) an ordinary $\mathrm{SM} 1 / \mathrm{HM}$ and (b) a SM1/Fe-Rh2/HM structure. A constant external field with $\mu_{0} H_{\text {ext }}=0.8 \mathrm{~T}$ in the up direction, as well as a heat pulse with $\sigma_{\text {pulse }}=140 \mathrm{ps}$ and $T_{\max }=500 \mathrm{~K}$, is assumed. (c) Switching time distribution obtained from 50000 switching trajectories, as displayed in (a) and (b), for three grain types.
TABLE VI. Switching-time distribution of various grain types. As illustrated in Fig. 9, the distribution for each grain type is obtained from 50000 switching trajectories with a pulse duration of $\sigma_{\text {pulse }}=140 \mathrm{ps}$ and a maximum pulse temperature of $T_{\max }=500 \mathrm{~K}$. A constant external field is assumed.

\begin{tabular}{lcc}
\hline \hline Grain & $r_{\text {ex }}(\%)$ & $\sigma_{\text {sw }}(\mathrm{ps})$ \\
\hline SM1/HM & $\ldots$ & 26.3 \\
SM1/Fe-Rh1/HM & 0 & 4.2 \\
SM1/Fe-Rh2/HM & 8 & 6.5 \\
\hline \hline
\end{tabular}

deviations of the obtained switching-time distributions are given in Table VI. The distribution decreases by a factor of 6 in the case of a SM1/Fe-Rh1/HM structure with $r_{\mathrm{ex}}=$ $0 \%$ and by a factor of 4 if $r_{\mathrm{ex}}=8 \%$ is assumed. The remaining ferromagnetic moment of the Fe-Rh interlayer does not influence the reduction much.

The reason for the narrowing of the switching-time distribution is displayed in Figs. 9(a) and 9(b), where one randomly chosen magnetization trajectory of the HM and SM1 layers is shown for both grain types. Without an $\mathrm{Fe}-\mathrm{Rh}$ interlayer, the SM1/HM grain has full exchange during the whole write process. The layers are strongly coupled at all times and switch together at $T \geq T_{f, \min }$. By contrast, the SM1 and the HM layer decouple for the external field used with a strength of $0.8 \mathrm{~T}$ at the beginning of the simulation for low temperatures. Since $H_{\mathrm{ext}}>$ $H_{\text {iex,SM1 }}$ is valid, the soft magnetic layer switches, whereas the hard magnetic layer does not, due to its high coercivity. Above $T_{\text {jump }}$, the exchange-spring effect reaches full strength. Hence, the SM1 layer can bias the HM-layer magnetization during switching, which significantly reduces the switching-time distribution.

The presented situation would occur for pulsed HAMR. In this technique, a laser spot moves over a recording medium and is switched on only at the desired write position; otherwise, it is switched off. To ensure high data rates, very short pulses with durations of about 100-200 ps are required. It is shown that, for such short pulses, dc noise is an issue for pure Fe-Pt-like grains [14] due to the short recording time window. The presented $\mathrm{SM} / \mathrm{Fe}-\mathrm{Rh} / \mathrm{HM}$ structure could solve this problem due to its reduced switching-time distribution. Because of the low heat-pulse temperatures, which are lower than the Curie temperature of all involved materials, all kinds of noise originating from thermal effects could be significantly reduced.

\section{CONCLUSION}

In this theoretical work, we extensively investigate the benefits and problems associated with an exchangecoupled structure with a high- and a low- $T_{C}$ part and an $\mathrm{Fe}-\mathrm{Rh}$ interlayer for heat-assisted magnetic recording (HAMR). The Fe-Rh layer, having a first-order phase 
transition from an antiferromagnetic phase at room temperature to a ferromagnetic phase at high temperatures, tunes the exchange coupling between a SM high- $T_{C}$ layer and a $\mathrm{HM}$ low- $T_{C}$ one. The coupling has its full strength at high temperatures, and at room temperature the exchange interactions reach their minimum. It is assumed that there remains a net ferromagnetic moment in the Fe-Rh layer even at low temperatures, which is large enough to stabilize the magnetization of the SM layer along the direction of the HM-layer magnetization, in order to achieve a high readback signal. We calculate footprints of a typical HAMR head on various grain types, with a coarse-grained LLB model. These footprints show that the transition jitter of the proposed $\mathrm{SM} / \mathrm{Fe}-\mathrm{Rh} / \mathrm{HM}$ structures is significantly reduced in both the down-track direction (by 20\%-30\%) and the off-track direction (by 30\%-50\%) compared to ordinary SM/HM grains. Additionally, because of the exchange-spring effect, no dc noise occurs-even at high head velocities and high write temperatures - in contrast to pure Fe-Pt grains. The origin for the transition jitter reduction is found to be a fast-increasing coercivity, or fast-increasing freezing temperature, during cooling of the $\mathrm{SM} / \mathrm{Fe}-\mathrm{Rh} / \mathrm{HM}$ structure.

Furthermore, a significant reduction of the switchingtime distribution of $\mathrm{SM} / \mathrm{Fe}-\mathrm{Rh} / \mathrm{HM}$ grains, by a factor of 4 , is found if short heat pulses, shorter than the write-field duration, are used. This reduction can be obtained if the maximum temperature of the heat pulse is smaller than the Curie temperature of the involved materials. The decrease of the switching-time distribution is based on a decoupling of the SM layer and the HM layer at room temperature, at which the Fe-Rh interlayer has a small or no net magnetic moment. Hence, the SM layer can independently switch in the write direction, and it thus can bias the HM layer at higher temperatures. This mechanism narrows the distribution of the switching times compared to ordinary $\mathrm{SM} / \mathrm{HM}$ grains.

The proposed grain with an Fe-Rh interlayer in the middle of the SM/HM structure seems to combine the best of pure HM and ordinary SM/HM grains. However, all presented results assume that a sufficiently thin Fe-Rh layer can be produced, which meets two basic requirements: (i) a net magnetic moment at room temperature, which is large enough to stabilize the SM layer but low enough that a pronounced phase transition is preserved, and (ii) a critical temperature of the phase transition which can be adapted. Individually, both properties seem to be physically possible, but it still requires some research effort to achieve both properties at the same time. This work demonstrates the benefits and chances of such a SM/Fe-Rh/HM structure. If requirement (i) is not obtainable, the presented results can still be instructive to explain the mechanisms how an $\mathrm{Fe}-\mathrm{Rh}$ capping layer in $\mathrm{Fe}-\mathrm{Rh} / \mathrm{HM}$ grains, as proposed in Ref. [16], can influence magnetic noise compared to pure Fe-Pt grains.

\section{ACKNOWLEDGMENTS}

The authors would like to thank the Vienna Science and Technology Fund (WWTF) under Grant No. MA14-044, the Advanced Storage Technology Consortium (ASTC), and the Austrian Science Fund (FWF) under Grant No. I2214-N20 for financial support. The computational results presented have been achieved using the Vienna Scientific Cluster (VSC).

[1] Roger Wood, The feasibility of magnetic recording at 1 terabit per square inch, IEEE Trans. Magn. 36, 36 (2000).

[2] Roger Wood, Mason Williams, Aleksandar Kavcic, and Jim Miles, The feasibility of magnetic recording at 10 terabits per square inch on conventional media, IEEE Trans. Magn. 45, 917 (2009).

[3] Dieter Weller, Oleksandr Mosendz, Gregory Parker, Simone Pisana, and Tiffany S. Santos, $\mathrm{L1}_{0}$ FePtX-Y media for heatassisted magnetic recording, Phys. Status Solidi (a) 210, 1245 (2013).

[4] Ludwig Mayer, Curie-point writing on magnetic films, J. Appl. Phys. 29, 1003 (1958).

[5] A. Q. Wu, Y. Kubota, T. Klemmer, T. Rausch, C. Peng, Y. Peng, D. Karns, X. Zhu, Y. Ding, E. K. C. Chang, Y. Zhao, H. Zhou, K. Gao, J. U. Thiele, M. Seigler, G. Ju, and E. Gage, HAMR areal density demonstration of $1+$ Tbpsi on spinstand, IEEE Trans. Magn. 49, 779 (2013).

[6] G. Ju, Y. Peng, E. Chang, Y. Ding, A. Wu, X. Zhu, Y. Kubota, T. Klemmer, H. Amini, L. Gao, Z. Fan, T. Rausch, M. Seigler, E. Gage, and J. Thiele, High density heat assisted magnetic recording media and advanced characterization-Progress and challenges, IEEE Trans. Magn. 51, 11 (2015).

[7] X. Wang, K. Gao, H. Zhou, A. Itagi, M. Seigler, and E. Gage, HAMR recording limitations and extendibility, IEEE Trans. Magn. 49, 686 (2013).

[8] D. Weller, G. Parker, O. Mosendz, E. Champion, B. Stipe, X. Wang, T. Klemmer, G. Ju, and A. Ajan, A HAMR media technology roadmap to an areal density of $4 \mathrm{~Tb} / \mathrm{in}^{2}$, IEEE Trans. Magn. 50, 1 (2014).

[9] Dieter Weller, Gregory Parker, Oleksandr Mosendz, Andreas Lyberatos, Dmitriy Mitin, Nataliia Y. Safonova, and Manfred Albrecht, Review article: FePt heat assisted magnetic recording media, J. Vac. Sci. Technol. B 34, 060801 (2016).

[10] Christoph Vogler, Claas Abert, Florian Bruckner, Dieter Suess, and Dirk Praetorius, Heat-assisted magnetic recording of bit-patterned media beyond $10 \mathrm{~Tb} / \mathrm{in}^{2}$, Appl. Phys. Lett. 108, 102406 (2016).

[11] Jian-Gang Zhu and Hai Li, Understanding signal and noise in heat assisted magnetic recording, IEEE Trans. Magn. 49, 765 (2013).

[12] Jian-Gang Zhu and Hai Li, Medium optimization for lowering head field and heating requirements in heat assisted magnetic recording, IEEE Magn. Lett. 6, 1 (2015). 
[13] Christoph Vogler, Claas Abert, Florian Bruckner, Dieter Suess, and Dirk Praetorius, Basic noise mechanisms of heat-assisted-magnetic recording, J. Appl. Phys. 120, 153901 (2016).

[14] Christoph Vogler, Claas Abert, Florian Bruckner, Dieter Suess, and Dirk Praetorius, Areal density optimizations for heat-assisted magnetic recording of high-density media, J. Appl. Phys. 119, 223903 (2016).

[15] Joseph Barker and Roy W. Chantrell, Higher-order exchange interactions leading to metamagnetism in FeRh, Phys. Rev. B 92, 094402 (2015).

[16] Jan-Ulrich Thiele, Stefan Maat, and Eric E. Fullerton, $\mathrm{FeRh} / \mathrm{FePt}$ exchange spring films for thermally assisted magnetic recording media, Appl. Phys. Lett. 82, 2859 (2003).

[17] Jian-Gang Zhu and David E. Laughlin, Binary anisotropy media, U.S. Patent No. US20080180827 (31 July 2008).

[18] T. J. Zhou, K. Cher, J. F. Hu, Z. M. Yuan, and B. Liu, The concept and fabrication of exchange switchable trilayer of $\mathrm{FePt} / \mathrm{FeRh} / \mathrm{FeCo}$ with reduced switching field, J. Appl. Phys. 111, 07C116 (2012).

[19] D. A. Garanin and O. Chubykalo-Fesenko, Thermal fluctuations and longitudinal relaxation of single-domain magnetic particles at elevated temperatures, Phys. Rev. B 70, 212409 (2004).

[20] O. Chubykalo-Fesenko, U. Nowak, R. W. Chantrell, and D. Garanin, Dynamic approach for micromagnetics close to the Curie temperature, Phys. Rev. B 74, 094436 (2006).

[21] U. Atxitia, O. Chubykalo-Fesenko, N. Kazantseva, D. Hinzke, U. Nowak, and R. W. Chantrell, Micromagnetic modeling of laser-induced magnetization dynamics using the Landau-Lifshitz-Bloch equation, Appl. Phys. Lett. 91, 232507 (2007).

[22] N. Kazantseva, D. Hinzke, U. Nowak, R. W. Chantrell, U. Atxitia, and O. Chubykalo-Fesenko, Towards multiscale modeling of magnetic materials: Simulations of FePt, Phys. Rev. B 77, 184428 (2008).

[23] C. Schieback, D. Hinzke, M. Kläui, U. Nowak, and P. Nielaba, Temperature dependence of the current-induced domain wall motion from a modified Landau-Lifshitz-Bloch equation, Phys. Rev. B 80, 214403 (2009).

[24] C. Bunce, J. Wu, G. Ju, B. Lu, D. Hinzke, N. Kazantseva, U. Nowak, and R. W. Chantrell, Laser-induced magnetization switching in films with perpendicular anisotropy: A comparison between measurements and a multi-macrospin model, Phys. Rev. B 81, 174428 (2010).

[25] J. Mendil, P. Nieves, O. Chubykalo-Fesenko, J. Walowski, T. Santos, S. Pisana, and M. Münzenberg, Resolving the role of femtosecond heated electrons in ultrafast spin dynamics, Sci. Rep. 4, 3980 (2014).

[26] U. Atxitia, D. Hinzke, and U. Nowak, Fundamentals and applications of the Landau-Lifshitz-Bloch equation, J. Phys. D 50, 033003 (2017).

[27] R. F. L. Evans, D. Hinzke, U. Atxitia, U. Nowak, R. W. Chantrell, and O. Chubykalo-Fesenko, Stochastic form of the Landau-Lifshitz-Bloch equation, Phys. Rev. B 85, 014433 (2012).

[28] Christoph Vogler, Claas Abert, Florian Bruckner, and Dieter Suess, Landau-Lifshitz-Bloch equation for exchangecoupled grains, Phys. Rev. B 90, 214431 (2014).
[29] W. A. Challener, Chubing Peng, A. V. Itagi, D. Karns, Wei Peng, Yingguo Peng, XiaoMin Yang, Xiaobin Zhu, N. J. Gokemeijer, Y.-T. Hsia, G. Ju, Robert E. Rottmayer, Michael A. Seigler, and E. C. Gage, Heat-assisted magnetic recording by a near-field transducer with efficient optical energy transfer, Nat. Photonics 3, 220 (2009).

[30] Nan Zhou, Xianfan Xu, Aaron T. Hammack, Barry C. Stipe, Kaizhong Gao, Werner Scholz, and Edward C. Gage, Plasmonic near-field transducer for heat-assisted magnetic recording, Nanophotonics 3, 141 (2014).

[31] J.-U. Thiele, K. R. Coffey, M. F. Toney, J. A. Hedstrom, and A. J. Kellock, Temperature dependent magnetic properties of highly chemically ordered $\mathrm{Fe}_{55-x} \mathrm{Ni}_{x} \mathrm{Pt}_{45} \mathrm{L1}_{0}$ films, J. Appl. Phys. 91, 6595 (2002).

[32] B. Wang and K. Barmak, Re-evaluation of the impact of ternary additions of $\mathrm{Ni}$ and $\mathrm{Cu}$ on the $\mathrm{A} 1$ to $\mathrm{L}_{0}$ transformation in FePt films, J. Appl. Phys. 109, 123916 (2011).

[33] Dustin A. Gilbert, Liang-Wei Wang, Timothy J. Klemmer, Jan-Ulrich Thiele, Chih-Huang Lai, and Kai Liu, Tuning magnetic anisotropy in (001) oriented $\mathrm{Ll}_{0}\left(\mathrm{Fe}_{1-x} \mathrm{Cu}_{x}\right)_{55} \mathrm{Pt}_{45}$ films, Appl. Phys. Lett. 102, 132406 (2013).

[34] W. Bailey, P. Kabos, F. Mancoff, and Stephen Russek, Control of magnetization dynamics in $\mathrm{Ni}_{81} \mathrm{Fe}_{19}$ thin films through the use of rare-earth dopants, IEEE Trans. Magn. 37, 1749 (2001).

[35] Claas Abert, Lukas Exl, Florian Bruckner, André Drews, and Dieter Suess, MAGNUM.FE: A micromagnetic finiteelement simulation code based on FENICS, J. Magn. Magn. Mater. 345, 29 (2013).

[36] D. R. Fredkin and T. R. Koehler, Hybrid method for computing demagnetizing fields, IEEE Trans. Magn. 26, 415 (1990).

[37] Y. Ohtani and I. Hatakeyama, Antiferro-ferromagnetic transition and microstructural properties in a sputter deposited FeRh thin film system, J. Appl. Phys. 74, 3328 (1993).

[38] Jiangwei Cao, Nguyen T. Nam, Sho Inoue, Hnin Yu Yu Ko, Nguyen N. Phuoc, and Takao Suzuki, Magnetization behaviors for FeRh single crystal thin films, J. Appl. Phys. 103, 07F501 (2008).

[39] Dhishan Kande, David Laughlin, and Jian-Gang Zhu, Origin of room temperature ferromagnetic moment in Rh-rich $[\mathrm{Rh} / \mathrm{Fe}]$ multilayer thin films, J. Appl. Phys. 107, 09E318 (2010).

[40] D. Kande, S. Pisana, D. Weller, D. E. Laughlin, and J. G. Zhu, Enhanced B2 ordering of FeRh thin films using B2 NiAl underlayers, IEEE Trans. Magn. 47, 3296 (2011).

[41] J. S. Kouvel, Unusual nature of the abrupt magnetic transition in FeRh and its pseudobinary variants, J. Appl. Phys. 37, 1257 (1966).

[42] C. W. Barton, T. A. Ostler, D. Huskisson, C. J. Kinane, S. J. Haigh, G. Hrkac, and T. Thomson, Substrate induced strain field in FeRh epilayers grown on single crystal $\mathrm{MgO}$ (001) substrates, Sci. Rep. 7, 44397 (2017).

[43] Thomas Andrew Ostler, Craig Barton, Thomas Thomson, and Gino Hrkac, Modeling the thickness dependence of the magnetic phase transition temperature in thin FeRh films, Phys. Rev. B 95, 064415 (2017). 
[44] Manish Kapoor, Xiao Shen, and R. H. Victora, Effect of intragranular exchange on exchange-coupled composite media, J. Appl. Phys. 99, 08Q902 (2006).

[45] D. Suess, J. Lee, J. Fidler, H. S. Jung, E. M. T. Velu, W. Jiang, S. S. Malhotra, G. Bertero, and T. Schrefl, Effect of intergranular exchange on the thermal stability and coercive field of perpendicular, single phase, exchange spring, and coupled granular continuous (CGC) perpendicular recording media, IEEE Trans. Magn. 45, 88 (2009).

[46] D. Suess, M. Fuger, C. Abert, F. Bruckner, and C. Vogler, Superior bit error rate and jitter due to improved switching field distribution in exchange spring magnetic recording media, Sci. Rep. 6, 27048 (2016). 